\title{
Search for streaming motion of galaxy clusters around the Giant Void ${ }^{\star}$
}

\author{
A. I. Kopylov ${ }^{1,2}$ and F. G. Kopylova ${ }^{1}$ \\ 1 Special Astrophysical Observatory of RAS, Nizhnij Arkhyz, Karachaevo-Cherkesia 369167, Russia \\ 2 Isaac Newton Institute, Chile, SAO Branch, Russia
}

Received 27 June 2001 / Accepted 27 September 2001

\begin{abstract}
We present the results of a study of streaming motion of galaxy clusters around the Giant Void $(\alpha \approx$ $13^{\mathrm{h}}, \delta \approx 40^{\circ}, z \approx 0.11$ and a diameter of $300 \mathrm{Mpc}$ ) in the distribution of rich Abell clusters. We used the Kormendy relation as a distance indicator taking into account galaxy luminosities. Observations were carried out in KronCousins $R_{\mathrm{c}}$ system on the $6 \mathrm{~m}$ and $1 \mathrm{~m}$ telescopes of SAO RAS. For 17 clusters in a spherical shell of $50 \mathrm{Mpc}$ in thickness centered on the void no significant diverging motion (expected to be generated by the mass deficit in the void) has been detected. This implies that cosmological models with low $\Omega_{\mathrm{m}}$ are preferred. To explain small mass underdensity inside the Giant Void, a mechanism of void formation with strong biasing is required.
\end{abstract}

Key words. galaxies: clusters - galaxies: elliptical and lenticular, $\mathrm{cD}$ - galaxies: fundamental parameters galaxies: photometry - galaxies: distances and redshifts - cosmology: large-scale structure of Universe

\section{Introduction}

To understand the origin and evolution of large scale structure in the universe it is important to study inhomogeneities of the largest size (mass) - superclusters and voids. Corresponding scales are 100-300 Mpc (here and further we use $H_{0}=50 \mathrm{~km} \mathrm{~s}^{-1} \mathrm{Mpc}^{-1}$ and $\left.q_{0}=0.5\right)$ and the mass excess (a deficit for voids) may reach $10^{16}$ $10^{17} M_{\odot}$. From a theoretical viewpoint the fact that voids might provide a key to understanding the large-scale structure of the Universe was emphasized by Zel'dovich et al. (1982).

The kinematical effect generated by inhomogeneities can be investigated by measurement of the peculiar (nonHubble) velocities of objects. Voids tend to expand at faster rates than the Hubble flow of the Universe, i.e., galaxies (clusters) on the edge of the void will have a peculiar velocity away from the centre of the void, matter within voids will have a tendency to be swept up into twodimensional sheets separating neighbouring voids. A large underdense volume may be modeled locally as a lower density Universe with a higher value of the local Hubble

Send offprint requests to: A. I. Kopylov, e-mail: akop@sao.ru

* Table 2 is only available in electronic form at the CDS via anonymous ftp to cdsarc.u-strasbg.fr (130.79.128.5) or via

http://cdsweb.u-strasbg.fr/cgi-bin/qcat?J/A+A/382/389 constant (within the underdense region) relative to the global value of the Hubble constant (Wu et al. 1996). The maximal peculiar velocity within the void is a rather sensitive function of $\Omega_{\mathrm{m}}$ and the density contrast in the void (Hoffman \& Shaham 1982; Wu et al. 1996). High velocity outflows are not expected in a low $\Omega$ universe. In particular, there is an $\Omega$-dependent limit on the magnitude of the diverging flow around a void: even a completely empty void cannot induce substantial peculiar motions if the mean density around it is well below the critical density (Dekel \& Rees 1994).

Extensive studies of the voids in the distribution of clusters which have catalogues of identified voids have been carried out by Batuski \& Burns (1985), Tully (1986), Einasto et al. (1994) and Stavrev (2000). One of the results of the programme "The Northern Cone of Metagalaxy" (Kopylov et al. 1988) was the discovery of the Giant Void $(\mathrm{GV})$ (with a size of $400 \mathrm{Mpc}$ and a redshift of the centre 0.116$)$ in the distribution of very rich $(R \geq 2)$ Abell clusters. Later a special programme to determine more precisely the extent of this void was undertaken (Kopylov 2001). On the $6 \mathrm{~m}$ telescope, redshifts were measured for clusters of richness $(R \geq 1)$, which constitute a statistical subsample of the Abell catalogue, in the direction of the GV. The size of the GV, defined as the size of largest empty sphere that can be accomodated inside the void, shrinked to $300 \mathrm{Mpc}$. Nevertheless, the volume of 400-500 Mpc in size remains sparsely populated by 
$R \geq 1$ Abell clusters, with a density of about $1 / 5$ of the global mean spatial density of such clusters. The GV has been also found by Stavrev (2000) as the largest void in the Northern Galactic Hemisphere (in the volume defined by galactic latitude $b \geq+30^{\circ}$ and redshift $z \leq 0.14$ ), with the largest void dimension $D_{\max }=550 \mathrm{Mpc}$ and the total volume of the void of $63.5 \times 10^{6} \mathrm{Mpc}^{3}$.

The search for streaming motion of galaxy clusters around the GV was undertaken as part of our long-term project started in 1993 to study peculiar motions of clusters induced by dark matter inhomogeneities on the scale of superclusters. For determination of cluster distances the Kormendy relation (Kormendy 1977) for early-type galaxies had been chosen as an economical distance estimator, based entirely on photometric parameters. Observational data have been obtained on the $6 \mathrm{~m}$ and $1 \mathrm{~m}$ telescopes of the Special Astrophysical Observatory of the Russian Academy of Sciences (SAO RAS) for the purpose of construction of a homogeneous photometric database for a large sample of early-type galaxies in clusters with redshifts $z=0.05-0.15$. In total, 38 clusters from the Ursa Major and Corona Borealis superclusters and in the spherical shell around the Giant Void were studied. Peculiar motions in two superclusters have been investigated in papers by Kopylova \& Kopylov (1998) and Kopylova \& Kopylov (2001a). The aim of the present paper is to investigate the velocity field around the Giant Void using a sample of 17 clusters.

In Sect. 2 we describe sample selection and in Sect. 3 our observations. Surface photometry of galaxies, internal and external comparisons of the measured parameters are described in Sect.4. Determination of parameters of the Kormendy relation, relative distances and peculiar velocities of clusters are presented in Sect. 5. Finally, conclusions are given in Sect. 6.

\section{Sample selection}

The diameter of the GV - the maximum diameter of a sphere devoid of clusters of richness $R \geq 1$ - is equal to $300 \mathrm{Mpc}$. Approximate coordinates of the void centre are $13^{\mathrm{h}} 01^{\mathrm{m}}+38^{\circ} 45^{\prime}$ (J2000). The redshift corresponding to the centre of the void is 0.107 . The clusters of galaxies in our sample are selected in a spherical shell centered on the GV, with an inner radius of $150 \mathrm{Mpc}$ and $50 \mathrm{Mpc}$ in thickness. Figure 1 shows the distribution of clusters in the neighbourhood of the void, projected on the plane passing across its centre. The diagram covers the region of space with coordinates: right ascension $10^{\mathrm{h}}-16^{\mathrm{h}}$, redshift $z<0.2$ and opening angle in declination $30^{\circ}(275 \mathrm{Mpc}$ at a distance of $600 \mathrm{Mpc}$, corresponding to GV centre). The rich clusters observed in the "Northern Cone" programme are shown by filled squares, other Abell clusters of richness $R \geq 1$ by filled triangles, and by open circles -17 clusters (all of richness $R=1$ ) at a distance of $150-200 \mathrm{Mpc}$ from the GV centre. The redshifts of 13 out of 17 clusters surrounding the void have been first measured by us. Three of them (A1361, A1637 and A1885) were later confirmed

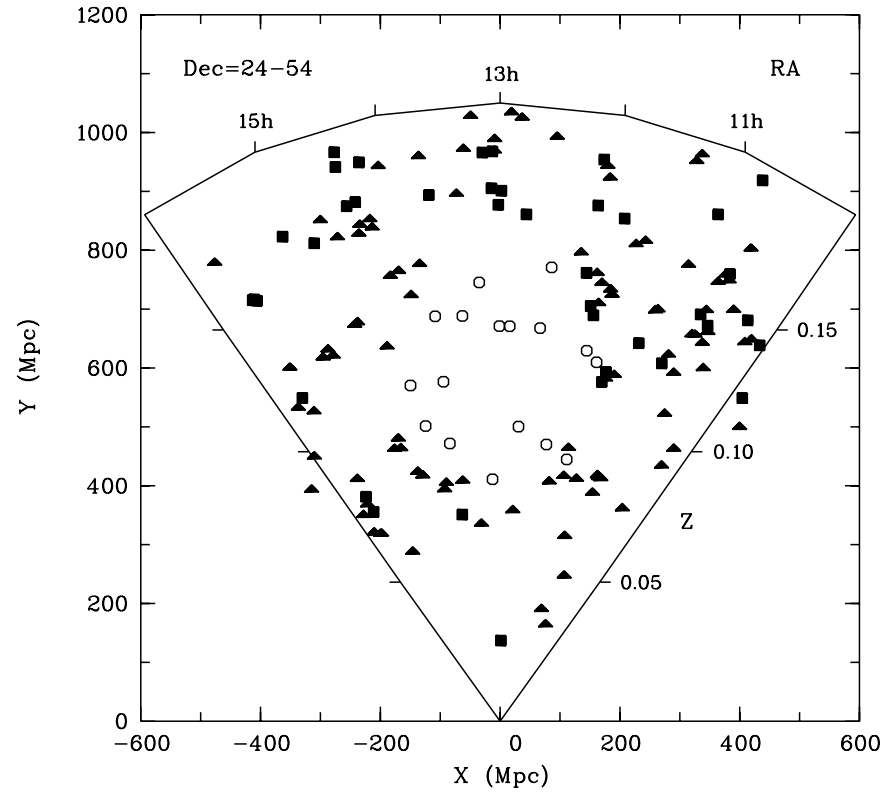

Fig. 1. Wedge diagram of the galaxy distribution around the Giant Void. "Northern Cone" rich clusters are plotted with filled squares, other Abell clusters $(R \geq 1)$ with filled triangles, GV clusters $(R=1)$ with open circles.

by other authors. Information on our sample of clusters is summarized in Table 1.

In order to derive the relative distances of clusters with the Kormendy relation, we have selected the brightest early-type galaxies for their apparently elliptical morphology on Palomar Sky Survey images, within 1.5-2 Mpc of the centre of the cluster. Although not all selected galaxies have measured redshifts to confirm their cluster membership, we assumed that the population of early-type galaxies in cluster cores has minimal contamination from field galaxies.

\section{Observations}

Observations were conducted on the $6 \mathrm{~m}$ and $1 \mathrm{~m}$ telescopes of SAO RAS in 1988-1998. The spectral observations were made on the $6 \mathrm{~m}$ telescope in 1988-89 and 1993 in IPCS mode (see the description of IPCS in Drabek et al. 1986 and Afanasiev et al. 1986) with the grating B1 (600 grooves $/ \mathrm{mm}$ ) that covers the range $3600-5500 \AA$ with a scale along the dispersion of $1.9 \AA$ /pixel. The accuracy of radial velocity measurements was usually equal to $100-200 \mathrm{~km} \mathrm{~s}^{-1}$.

The photometric observations were carried out with a standard Kron-Cousins $R_{\mathrm{c}}$ filter at the prime focus of the $6 \mathrm{~m}$ telescope during observing runs on February and April 1994 and on the $1 \mathrm{~m}$ telescope (Zeiss-1000) on March 1995 and April 1998. The CCD photometer at the $6 \mathrm{~m}$ telescope had a chip "ISD015A" of $520 \times 580$ pixels, each $18 \times 24 \mu \mathrm{m}$, which corresponds to an angular size $0.154^{\prime \prime} \times 0.205^{\prime \prime}$. At the $1 \mathrm{~m}$ telescope the CCD photometer has an analogous chip with pixel size of $0.28^{\prime \prime} \times 0.37^{\prime \prime}$. The exposure time was usually $200 \mathrm{~s}$ on the $6 \mathrm{~m}$ telescope and $400-600 \mathrm{~s}$ on the 
Table 1. Cluster data. Column 1 gives the Abell catalogue number. Columns 2 and 3 give Right Ascension and Declination (equinox J2000) of the cluster centre. Columns 4-6 show Abell richness count, Bautz-Morgan type and redshift (corrected for the solar motion, $\Delta z=0.001 \sin (l) \cos (b))$, respectively. Column 7 gives number of galaxies with measured redshift. Column 8 redshift references.

\begin{tabular}{|c|c|c|c|c|c|c|c|}
\hline $\begin{array}{c}\text { Abell \# } \\
(1)\end{array}$ & $\begin{array}{l}\text { RA } \\
(2)\end{array}$ & $\begin{array}{c}\text { Dec } \\
(3)\end{array}$ & $\begin{array}{l}N_{\mathrm{A}} \\
(4)\end{array}$ & $\begin{array}{l}\mathrm{BM} \\
(5)\end{array}$ & $\begin{array}{c}z \\
(6)\end{array}$ & $\begin{array}{l}n_{z} \\
(7)\end{array}$ & $\begin{array}{l}\operatorname{Ref}^{c} \\
(8)\end{array}$ \\
\hline A1298 & 1132.5 & +4448 & 63 & II-III & 0.1154 & 2 & 1 \\
\hline A1361 & 1143.8 & +4621 & 57 & I-II & $0.1171^{b}$ & 3 & $1,2,3,11,12,13$ \\
\hline A1427 & 1158.4 & +3042 & 68 & II-III & 0.0810 & 2 & 1 \\
\hline A1468 & 1205.6 & +5125 & 50 & I: & 0.0847 & 6 & 4 \\
\hline A1542 & 1227.6 & +4926 & 73 & II-III & 0.1218 & 2 & 1 \\
\hline A1551 & 1229.7 & +3639 & 50 & III & 0.1449 & 1 & 1 \\
\hline A1609 & 1246.5 & +2625 & 56 & II-III: & 0.0882 & 2 & $1,5,6,11$ \\
\hline A1637 & 1253.9 & +5048 & 60 & III & 0.1220 & 2 & 1,7 \\
\hline $\mathrm{A} 1666^{a}$ & 1303.1 & +5116 & 54 & III & 0.1221 & 4 & 1 \\
\hline A1691 & 1311.4 & +3912 & 64 & II & 0.0724 & 70 & 8,9 \\
\hline A 1700 & 1314.7 & +2843 & 58 & III: & 0.1388 & 2 & 1 \\
\hline A1739 & 1326.2 & +2926 & 51 & III & 0.1266 & 3 & 1 \\
\hline A1793 & 1348.3 & +3217 & 54 & III & 0.0838 & 8 & 9 \\
\hline A1823 & 1356.7 & +4455 & 52 & II-III: & 0.1284 & 2 & 1 \\
\hline A1834 & 1358.7 & +4932 & 52 & III & 0.1062 & 3 & 1 \\
\hline A1885 & 1413.8 & +4340 & 63 & II-III & 0.0906 & 4 & 1,10 \\
\hline A1894 & 1417.7 & +4322 & 60 & III & 0.1069 & 2 & 1 \\
\hline
\end{tabular}

${ }^{a}$ We identify this cluster with the Zwicky cluster J1303.7+5118 as at the Abell cluster coordinates (J1302.8+5153) none rich cluster was found. Coordinates for the main concentration of galaxies are given.

${ }^{b}$ Redshift of strongly dominated cD galaxy is given.

${ }^{c}$ Redshift references: (1) this paper, (2) Allen et al. (1992), (3) Schneider et al. (1994), (4) Maurogordato et al. (1997), (5) Fanti et al. (1978), (6) Barton et al. (1998), (7) Laurent-Muehleisen et al. (1998), (8) Hill \& Oegerle (1998), (9) Slinglend et al. (1998), (10) Crawford et al. (1995), (11) Owen et al. (1995), (12) Cao et al. (1999), (13) Kim \& Elvis (1999).

$1 \mathrm{~m}$ telescope. The seeing (measured as the $F W H M$ of a stellar profile) during the observations was better than $2^{\prime \prime}$ with an average of $1^{\prime \prime} 4$. We tried to observe more distant clusters under the best seeing conditions $\left(\approx 1^{\prime \prime}\right)$.

Photometric standards from Landolt (1992) were observed in all runs to transform instrumental magnitudes into the standard $R_{\mathrm{c}}$ system. To correct a variable extinction that was present during observations on the $6 \mathrm{~m}$ telescope, all galaxies were exposed once more on the $1 \mathrm{~m}$ telescope under good photometric conditions in March 1995 and April 1998. Four clusters (A1361, A1691, A1834 and A1885) were observed on the $1 \mathrm{~m}$ telescope only. The observations with the $1 \mathrm{~m}$ telescope allowed us to bring almost all data to a common photometric scale with an accuracy of no worth than $0 .{ }^{\mathrm{m}} 03$.

The photometric processing of the frames was performed with the PC VISTA (Treffers \& Richmond 1989) and ESO MIDAS (ESO 1994) packages. We applied a standard procedure for reducing observational data: dark frame subtraction, flat-fielding and cleaning of cosmic events.

\section{Surface photometry}

We conducted integrated photometry in increasing circular apertures (with 1 or 2 pixel increment in radius) from a prechosen center to faint outskirts of galaxies in order to estimate total magnitudes and parameters of the surface brightness profiles. The total magnitude $m_{R}$ of a galaxy was estimated as an asymptotic value of the obtained radial growth curve. The residual mean error in the measured background level determined by using several areas in a frame was better than $0.5 \%$. Contaminating objects (stars, galaxies) were removed. We applied a procedure according to which the area of the contaminating object was replaced by a "clear area" located symmetrically about the centre of the galaxy.

The growth curve was used to determine the effective radius $r_{\mathrm{e}}$ at which the relation $m_{R}\left(r_{\mathrm{e}}\right)=m_{R}+0$. 75 is satisfied, as well as the effective brightness $\mu_{\mathrm{e}}$ at this radius and average effective surface brightness $\left\langle\mu_{\mathrm{e}}>\right.$ within this radius. We have corrected $r_{\mathrm{e}}, \mu_{\mathrm{e}}$ and $\left\langle\mu_{\mathrm{e}}\right\rangle$ for the effect of seeing following Saglia et al. (1993). The measured photometric parameters and equatorial coordinates 
of 210 galaxies are listed in Table 2. It has the following contents: Col. 1 - Abell catalogue number of the cluster; Col. 2 - identification number of the galaxy; Cols. 3 and 4 - equatorial coordinates (Right Ascension and Declination, J2000) measured on DSS2 plates; Col. 5 - total (asymptotic) $R_{\mathrm{c}}$ magnitude corrected for Galactic extinction (value of extinction corresponds to the centre of cluster and was taken from the NED, where the data of Schlegel et al. (1998) were used); Col. 6 - effective radius in arcseconds; Col. 7 - effective surface brightness in mag $\operatorname{arcsec}^{-2}$; Col. 8 - average effective surface brightness in mag $\operatorname{arcsec}^{-2}$ (though not used in this paper it is given for completeness); Col. 9 - 33 galaxies excluded from the analysis are marked: if fainter than $M_{R} \approx-21.5-1$; disk dominated -2 ; peculiar or interacting -3 .

By comparing repeated observations of 36 galaxies we evaluate the internal consistency of our photometric measurements. The total magnitudes have a rms scatter of 0 . 051 , the effective radii of $0.037 \mathrm{dex}$, the effective surface brightnesses of $0.118 \mathrm{mag} \mathrm{arcsec}^{-2}$.

No data set of $R$-band photometric observations of early-type galaxies of these clusters is available for an external comparison of our photometric measurements. An indirect test can be obtained by comparison with similar measurements presented by Saglia et al. (1997) (EFAR project) for galaxies in the Coma cluster. We have measurements of 19 galaxies in common with the sample of Saglia et al. (1997). There are no significant offsets between our values and EFAR values; specifically, $\Delta m_{\text {tot }}=-0.002$ with an rms of $0 .{ }^{\mathrm{m}} 164, \Delta \log r_{\mathrm{e}}=-0.013$ with rms of 0.107 dex and $\Delta<\mu_{\mathrm{e}}>=-0.078$ with rms of $0.394 \mathrm{mag} \mathrm{arcsec}^{-2}$.

\section{Distances and peculiar velocities}

In order to determine relative distances and peculiar velocities of clusters, we used the projection of the Fundamental Plane (FP) of early-type galaxies (Dressler et al. 1987; Djorgovski \& Davis 1987) onto the plane defined by $R_{\mathrm{e}}$ and $\mu_{\mathrm{e}}$, the Kormendy relation $(K R)$ (Kormendy 1977), in the form where the effective radius $R_{\mathrm{e}}$ is given as a function of effective surface brightness $\mu_{\mathrm{e}}: \log \left(R_{\mathrm{e}}\right)=A \mu_{\mathrm{e}}+C$. Then individual cluster zero-points $C$ are used to derive relative distances.

\subsection{The Kormendy relation for clusters}

Figure 2 presents the Kormendy diagram for our combined sample of the GV, the Ursa Major and the Corona Borealis superclusters, and the Coma cluster: $434 \mathrm{E}$ and S0 galaxies in total. Parameters of these galaxies have been reduced to the comoving system of distances by applying to measured values the cosmological corrections which correspond to individual cluster redshifts. Relativistic effects and changes in the geometry of the Universe contribute a correction $\Delta \mu_{\mathrm{e}}=10 \log \left(1+z_{\text {spec }}\right)$, where $z_{\text {spec }}$ is the mean cluster redshift which includes the peculiar velocity. We applied the $K$-correction in the form $K_{R}(z)=1.1 z$.

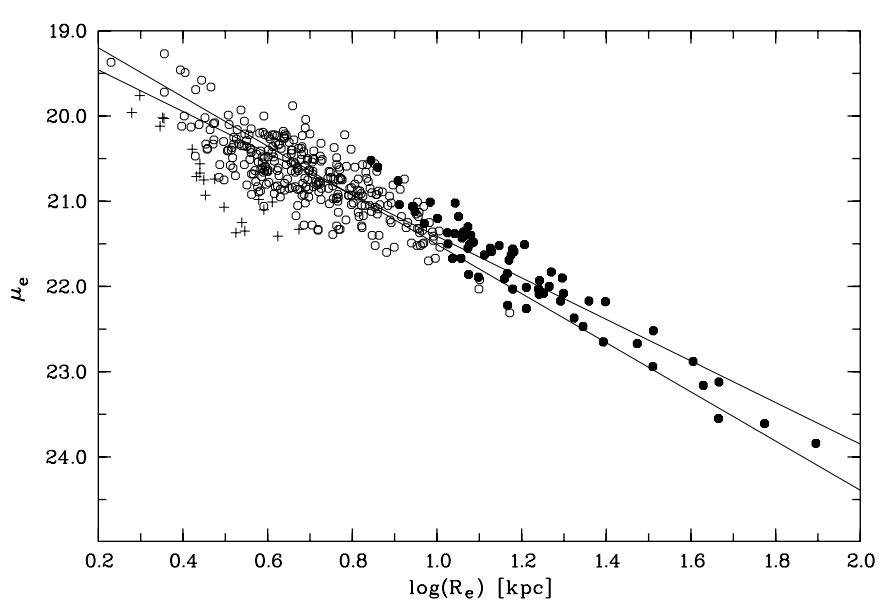

Fig. 2. The Kormendy relation for 434 early-type galaxies in 39 clusters subdivided into three magnitude-bins: $M_{R}<$ $-23^{\mathrm{m}} 5$ (filled circles), $-21^{\mathrm{m}} 5<M_{R}<-23^{\mathrm{m}} 5$ (open circles), $M_{R}>-21^{\mathrm{m}} 5$ (pluses). The lines are least-square fits with variables interchanged.

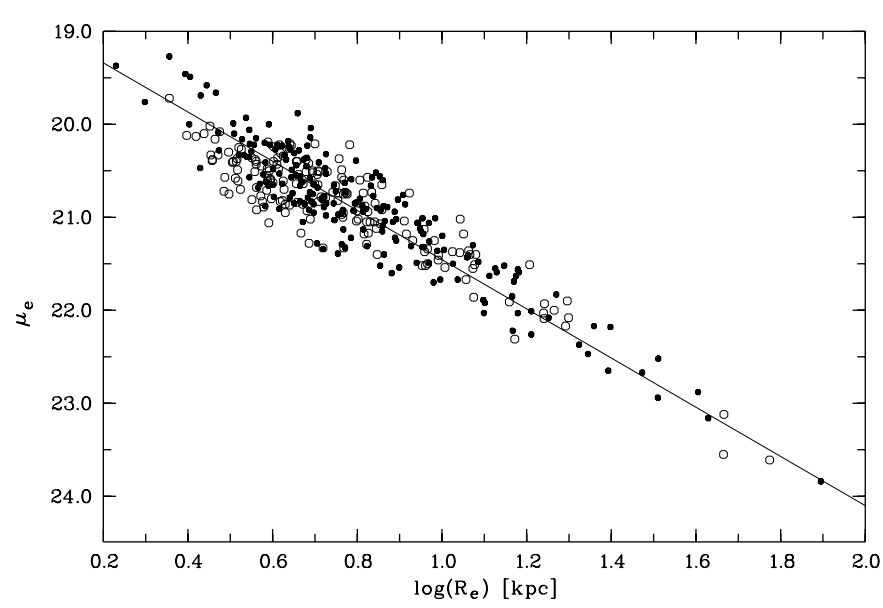

Fig. 3. The Kormendy relation for Giant Void clusters (open circles) at the background of rest of the total sample (filled circles). The line is an average of forward and inverse leastsquare fits.

The angular radius $r_{\mathrm{e}}$ was converted to the linear radius $R_{\mathrm{e}}$ in kiloparsecs. Thus, all data in Fig. 2 are shown in a comoving coordinate system for a "standard cosmological model" $\left(H_{0}=50 \mathrm{~km} \mathrm{~s}^{-1} \mathrm{Mpc}^{-1}\right.$ and $\left.q_{0}=0.5\right)$.

In Table 3 we summarize the fit parameters of the $K R$ for various limiting magnitudes and various samples. The slope $A$ and zero-point $C$ of the $K R$ are calculated as average values of forward and inverse least-squares fits that are given in brackets. Four upper lines of Table 3 show that the slope $A$ and zero-point $C$ of the $K R$ depend on the limiting magnitude of the sample. Figure 2 illustrates this fact: galaxies with $M_{R}>-21.5$ (pluses) strongly deviate from the $K R$.

For comparison in Table 3 the slope $A$ and zero-point $C$ from our determination are given for the Coma cluster (Kopylova \& Kopylov 2001b) and EFAR early-type galaxies in Abell clusters (Saglia et al. 1997). For the 


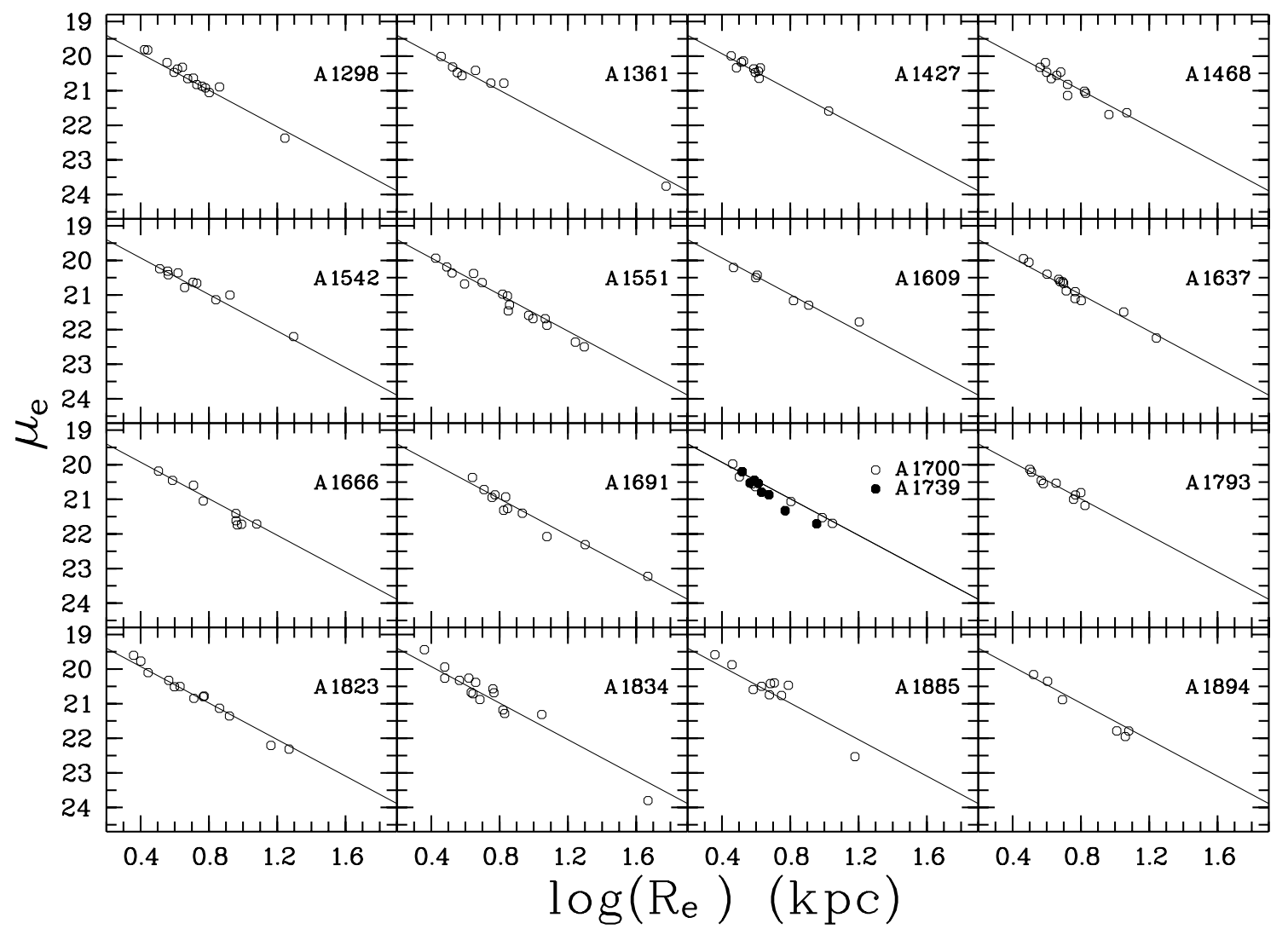

Fig. 4. The magnitude-corrected Kormendy relations for 17 clusters. The line corresponds to the average zero-point of these clusters.

Coma cluster the classification of galaxies was taken from Dressler (1980). The good consistency of the slopes and zero-points is evaluated through the comparison of our $M_{R} \leq-21.5$ sample and the EFAR sample.

In order to derive the relative distances of clusters with $K R$, we assumed that the slope of the $K R$ is the same for all clusters. Galaxies were selected to a limiting absolute magnitude $M_{R}=-21.5$. For this limit we used $A=0.3786, C=-7.123$. Figure 3 shows the $K R$ for $\mathrm{GV}$ clusters (open circles) on the background of the rest of our total sample (filled circles). The line is an average of forward and inverse least-square fits. On this diagram the $K R$ has an average scatter which is equivalent to a $24 \%$ uncertainty on the distance to a single galaxy.

The residuals of $K R$ calculated as $\Delta K R=\log \left(R_{\mathrm{e}}\right)-$ $0.3786 \mu_{\mathrm{e}}+7.123$ are correlated with the galaxy luminosity (Gudehus 1991; Scodeggio et al. 1997), and followed approximately a quadratic relation. This effect makes the $K R$ very sensitive to the cluster population incompleteness. The least-squares fit of a second-order polynomial to the $\Delta K R-M_{R}$ relation for our data is given by $\Delta K R=$ $A_{0}+A_{1} M_{R}+A_{2} M_{R}^{2}$, where $A_{0}=-23.949609, A_{1}=$ $-2.001820, A_{2}=-0.041696$. Subtracting a term $\left(A_{0}+\right.$ $\left.A_{1} M_{R}+A_{2} M_{R}^{2}\right) / 0.3786$ from the $\mu_{\mathrm{e}}$ for each galaxy, the corrected KR is obtained, which gives a $15 \%$ uncertainty on the distance to a single galaxy.
Figure 4 shows individual Kormendy diagrams for 17 clusters where the measured $\mu_{\mathrm{e}}$ is corrected for the absolute magnitude of each galaxy by subtracting the term $\left(A_{0}+A_{1} M_{R}+A_{2} M_{R}^{2}\right) / 0.3786$. The KR with a zero-point, which is the mean for these 17 clusters, is shown with a line for comparison. The mean statistical error of the cluster distance, when averaging 6-14 galaxies, is about $4 \%$.

\subsection{Peculiar velocities}

The differences between the zero-point for each cluster $C_{\mathrm{cl}}$ and the mean zero-point for all clusters $C_{\mathrm{m}}$ are used to determine photometric redshifts (distances) of clusters: $z_{\text {phot }}=z_{\text {spec }} 10^{\left(C_{\mathrm{m}}-C_{\mathrm{cl}}\right)}$. We derived the peculiar velocities of clusters in a comoving system from the difference between spectroscopic and photometric redshifts using the formula $V_{\text {pec }}=c\left(z_{\text {spec }}-z_{\text {phot }}\right) /\left(1+z_{\text {phot }}\right)$.

We have compared the precision of our method with respect to the FP method. Figure 5 shows good agreement between the peculiar velocities measured applying our modification of the $K R$ method to EFAR data (Abell clusters from Table 3 of Colless et al. 2000) and the FP estimates (from Table 7 of Colless et al. 2000). The correlation coefficient is equal to 0.70 for all 22 clusters and 0.82 if we exclude the two most deviating clusters (A419 and A548-2). 
Table 3. Determinations of the Kormendy relation parameters. The second column gives the number of galaxies of the respective sample. $A$ and $C$ are slope and zero-point of the average fit to the respective Kormendy relation. The values in brackets are $A$ and $C$ of the forward and inverse least-squares fits.

\begin{tabular}{lrcc}
\hline sample & $N$ & $A$ & $C$ \\
\hline 39 clusters $\left(M_{R} \leq-24.5\right)$ & 10 & $0.28(0.27 ; 0.29)$ & $-4.90(-4.66 ;-5.14)$ \\
39 clusters $\left(M_{R} \leq-23.5\right)$ & 66 & $0.32(0.31 ; 0.33)$ & $-5.76(-5.49 ;-6.02)$ \\
39 clusters $\left(M_{R} \leq-22.5\right)$ & 240 & $0.34(0.33 ; 0.36)$ & $-6.40(-6.04 ;-6.76)$ \\
39 clusters $\left(M_{R} \leq-21.5\right)$ & 381 & $0.38(0.35 ; 0.41)$ & $-7.12(-6.46 ;-7.79)$ \\
Coma $(\mathrm{E})\left(M_{R} \leq-21.5\right)$ & 25 & $0.37(0.36 ; 0.38)$ & $-7.00(-6.78 ;-7.22)$ \\
Coma $(\mathrm{E}+\mathrm{SO})\left(M_{R} \leq-21.5\right)$ & 38 & $0.35(0.33 ; 0.38)$ & $-6.64(-6.10 ;-7.18)$ \\
EFAR $(\mathrm{E}+\mathrm{E} / \mathrm{SO})\left(M_{R} \leq-21.5\right)$ & 186 & $0.37(0.34 ; 0.41)$ & $-7.03(-6.25 ;-7.81)$ \\
\hline
\end{tabular}

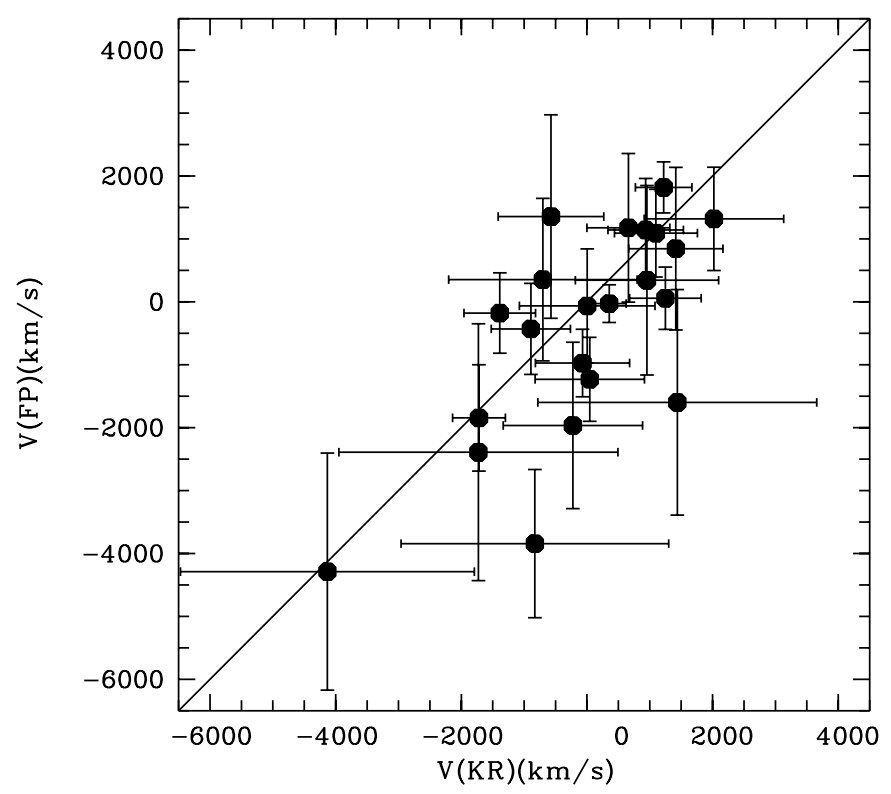

Fig. 5. For 22 clusters of the EFAR project peculiar velocities measured using the FP in Colless et al. (2000) are compared with those measured by means of the KR with correction for absolute magnitude of the galaxy.

The results of our measurements of photometric distances and peculiar velocities of 17 clusters are presented in Table 4, which have the following contents: Col. 1 Abell catalogue number; Col. 2 - number of galaxies used; Col. 3 - zero-point of the $K R$ fit, $C_{\mathrm{cl}}$, with its uncertainty; Col. 4 - redshift $z_{\text {phot }}$ which corresponds to the photometric distance $C_{\mathrm{cl}}$; Cols. 5, 6 - peculiar velocity and its uncertainty $\left(\mathrm{km} \mathrm{s}^{-1}\right)$, respectively.

\subsection{Outflow from the Giant Void}

The distribution of the clusters in the redshift - zeropoint diagram (zero-points correspond here to distances in the comoving system) is shown in Fig. 6 without (on the left) and with (on the right) correction for luminosity evolution according to the model for elliptical galaxies of
Table 4. Distances and peculiar velocities.

\begin{tabular}{crccrr}
\hline Abell \# & $N$ & $C_{c l}$ & $z_{\text {phot }}$ & $V_{\text {pec }}$ & $\delta V_{\text {pec }}$ \\
1 & 2 & 3 & 4 & 5 & 6 \\
\hline A1298 & 14 & $-7.116 \pm 0.014$ & 0.1074 & 2160 & 920 \\
A1361 & 8 & $-7.142 \pm 0.024$ & 0.1157 & 370 & 1750 \\
A1427 & 10 & $-7.141 \pm 0.013$ & 0.0798 & 320 & 690 \\
A1468 & 12 & $-7.150 \pm 0.019$ & 0.0852 & -140 & 1030 \\
A1542 & 10 & $-7.125 \pm 0.016$ & 0.1158 & 1620 & 1160 \\
A1551 & 16 & $-7.176 \pm 0.014$ & 0.1547 & -2540 & 1300 \\
A1609 & 6 & $-7.142 \pm 0.022$ & 0.0871 & 300 & 1740 \\
A1637 & 13 & $-7.140 \pm 0.013$ & 0.1200 & 520 & 960 \\
A1666 & 9 & $-7.175 \pm 0.019$ & 0.1303 & -2170 & 1510 \\
A1691 & 11 & $-7.160 \pm 0.019$ & 0.0745 & -590 & 920 \\
A1700 & 7 & $-7.174 \pm 0.015$ & 0.1478 & -2350 & 1300 \\
A1739 & 8 & $-7.210 \pm 0.021$ & 0.1462 & -5140 & 1870 \\
A1793 & 9 & $-7.151 \pm 0.014$ & 0.0846 & -220 & 760 \\
A1823 & 13 & $-7.141 \pm 0.014$ & 0.1266 & 480 & 1050 \\
A1834 & 15 & $-7.133 \pm 0.026$ & 0.1029 & 900 & 1660 \\
A1885 & 10 & $-7.108 \pm 0.032$ & 0.0829 & 2130 & 1710 \\
A1894 & 6 & $-7.179 \pm 0.026$ & 0.1152 & -2230 & 1890 \\
\hline
\end{tabular}

Poggianti (1997). For our range of redshifts this correction can be approximated by the relation $E_{R}(z)=-1.2 z$.

As seen in Figs. 1 and 6, it is possible to divide data into two subsamples of clusters: A1427, A1468, A1609, A1793, A1885 at the low-velocity and A1298, A1361, A1542, A1637, A1666, A1823 at the high-velocity boundaries (shells) of the GV. The average redshifts of these boundaries are 0.0857 and 0.1211 . Three open circles in Fig. 6 correspond to strongly deviating clusters. We suppose that two of these clusters, A1551 and A1700, are located farther than the high-velocity edge of the GV. The most deviating A1739 may be more distant than its measured redshift indicates. As this cluster is of distance class 6 in the Abell catalogue, its redshift could be related to a foreground poor cluster or group of galaxies projected on a more distant cluster. 


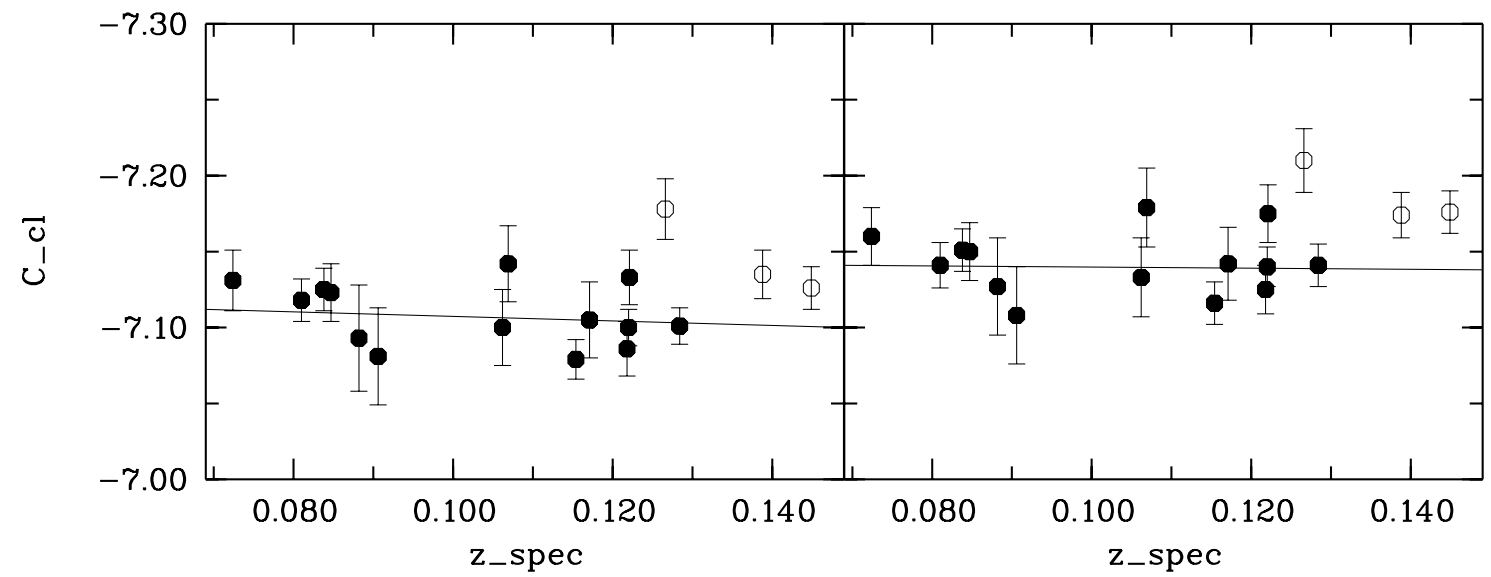

Fig. 6. Relative distances as a function of redshift for 17 clusters around the Giant Void without (on the left) and with (on the right) correction for luminosity evolution. Error bars are $\sigma / \sqrt{(} N-1)$. The lines show the linear regressions for clusters that define the high- and low-velocity boundaries of the GV (see text). The most deviating clusters (A1551, A1700, A1739) are marked by open circles.

The lines in Fig. 6 correspond to the linear regressions determined using the clusters at high- and low-velocity boundaries: $C_{\mathrm{cl}}=0.317( \pm 0.30) z-7.139( \pm 0.02)$ (on the left) and $C_{\mathrm{cl}}=0.033( \pm 0.32) z-7.166( \pm 0.02)$ (on the right). The corresponding peculiar outflow velocities are $450 \pm 440 \mathrm{~km} \mathrm{~s}^{-1}$ (on the left) and $50 \pm 450 \mathrm{~km} \mathrm{~s}^{-1}$ (on the right). The data are consistent with the negligible outflow from the GV in the case where the correction for luminosity evolution of galaxies was applied.

\section{Conclusions}

We have measured photometric distances and peculiar velocities for 17 clusters of galaxies, with redshifts between 0.07 and 0.15 , distributed in the shell around the Giant Void. Velocities were determined by the Kormendy relation distance estimates for early-type galaxies. We use the average least-squares fit to the 381 galaxies $\left(M_{R}<-21^{\mathrm{m}} 5\right)$ to fix the slope of the Kormendy relation. Nonlinearity of the Kormendy relation has been corrected by the second-order polynomial relation between the residuals and absolute magnitudes of galaxies. We demonstrate (Fig. 5) that peculiar velocities obtained using this corrected relation are consistent with those measured by the FP method for the subsample of EFAR clusters (Colless et al. 2000).

We have not found the outflow of clusters from the Giant Void. The $1 \sigma$ upper limit of $500 \mathrm{~km} \mathrm{~s}^{-1}$ has been obtained to the expansion velocity of this underdense region. The ratio of the outflow velocity to the Hubble flow velocity across the GV is smaller than 0.05. As the amplitude of outflow constrains a combination of the matter density contrast and the cosmological density parameter $\Omega_{\mathrm{m}}$ (Hoffman \& Shaham 1982), our result implies that cosmological models with small $\Omega_{\mathrm{m}}$ are preferred. But even for the usually adopted $\Omega_{\mathrm{m}} \approx 0.25$, the mass underdensity inside the GV should be much smaller than that inferred from the distribution of rich clusters of galaxies. This means that a purely gravitational formation mechanism may be insufficient for giant voids and more complex models of void formation, e.g. with strong biasing (Friedmann \& Piran 2001), should be invoked.

Acknowledgements. This work has been supported by the Russian Foundation for Basic Research, project No. 93-0217186. The Digitized Sky Surveys were produced at the Space Telescope Science Institute under U.S. Government grant NAG W-2166. This research has made use of the NASA/IPAC Extragalactic Database (NED) which is operated by the Jet Propulsion Laboratory, California Institute of Technology, under contract with the National Aeronautics and Space Administration.

\section{References}

Abell, G. O., Corwin, Jr. H. G., \& Olowin, R. P. 1989, ApJS, 70,1

Afanasiev, V. L., Lipovetsky, V. A., Mikhailov, V. P., \& Nazarov, E. A. 1986, Report SAO No. 138

Allen, S. W., Edge, A. C., Fabian, A. C., et al. 1992, MNRAS, 259,67

Barton, E. J., de Carvalho, R. R., \& Geller, M. J. 1998, AJ, 116,1573

Batuski, D. J., \& Burns, J. O. 1985, AJ, 90, 1413

Cao, L., Wei, J.-Y., \& Hu, J.-Y. 1999, A\&A, 135, 243

Colless, M., Saglia, R. P., Burstein, D., et al. 2001, MNRAS, 321,277

Crawford, C. S., Edge, A. C., Fabian, A. C., Allen, S. W., et al. 1995, MNRAS, 274, 75

Dekel, A., \& Rees, M. J. 1994, ApJ, 422, L1

Djorgovski, S., \& Davis, M. 1987, ApJ, 313, 59

Drabek, S. V., Kopylov, I. M., Somov, N. N., \& Somova, T. A. 1986, Astrophyz. Issled., 22, 64

Dressler, A. 1980, ApJS, 42, 565

Dressler, A., Lynden-Bell, D., Burstein, D., et al. 1987, ApJ, 313,42 
Einasto, M., Einasto, J., Tago, E., Dalton, G. B., \& Andernach, H. 1994, MNRAS, 269, 301

ESO 1994, MIDAS manual, European Southern Observatory

Fanti, R., Gioia, I., Lari, C., \& Ulrich, M. H. 1978, A\&A, 34, 341

Friedmann, Y., \& Piran, T. 2001, ApJ, 548, 1

Gudehus, D. H. 1991, ApJ, 382, 1

Hill, J. M., \& Oegerle, W. R. 1998, AJ, 116, 1529

Hoffman, Y., \& Shaham, J. 1982, ApJ, 262, L23

Kim, D.-W., \& Elvis, M. 1999, ApJ, 516, 9

Kopylov, A. I. 2001, in preparation

Kopylov, A. I., Kuznetsov, D. Yu., Fetisova, T. S., \& Shvartsman, V. F. 1988, in Large Scale Structures of the Universe, ed. Audouse J. et al., 129

Kopylova, F. G., \& Kopylov, A. I. 1998, Astro. Lett., 24, 291

Kopylova, F. G., \& Kopylov, A. I. 2001a, Astro. Lett., 27, 345

Kopylova, F. G., \& Kopylov, A. I. 2001b, in preparation

Kormendy, J. 1977, ApJ, 218, 333

Landolt, A. U. 1992, AJ, 104, 340

Laurent-Muehleisen, S. A., Kollgaard, R. I., Ciardullo, R., et al. 1998, ApJS, 118, 127
Maurogordato, S., Proust, D., Cappi, A., et al. 1997, A\&A, 123,411

Owen, F. N., Ledlow, M. J., \& Kell, W. C. 1995, AJ, 109, 140

Poggianti, B. M. 1997, A\&A, 122, 399

Saglia, R. P., Bertschinger, E., Baggley, G., et al. 1993, MNRAS, 264, 961

Saglia, R. P., Burstein, D., Baggley, G., et al. 1997, MNRAS, 292, 499

Schlegel, D. J., Finkbeiner, D. P., \& Davis, M. 1998, ApJ, 500, 525

Schneider, D. P., Schmidt, M., \& Gunn, J. E. 1994, AJ, 107, 1245

Scodeggio, M., Giovanelli, R., \& Haynes, M. 1997, AJ, 113, 208

Slinglend, K., Batuski, D., Miller, C., et al. 1998, ApJS, 115, 1

Stavrev, K. Y. 2000, A\&A, 144, 323

Tully, R. B. 1986, ApJ, 303, 25

Treffers, R. R., \& Richmond M. W., 1989, PASP, 101, 725

Wu, X.-P., Qin, B., \& Fang, L.-Z. 1996, ApJ, 469, 48

Zel'dovich, Ya. B., Einasto, J., \& Shandarin, S. F. 1982, Nature, 300, 407 